\title{
Black Holes, Gravitational Waves and Space Time Singularities
}

\author{
Stefano Bellucci ${ }^{1}$ - Alfio Bonanno ${ }^{2,3}$. Gabriele Gionti S. J.,4,5 \\ Fabio Scardigli ${ }^{6}$
}

Published online: 1 August 2018

๑) Springer Science+Business Media, LLC, part of Springer Nature 2018

This special issue of Foundations of Physics addresses the exciting topics of Black Holes, Gravitational Waves, and Space Time Singularities, in a compilation that includes articles by several of the pioneers in these fields.

Today, everybody knows that the Universe in which we live began in a "hot phase" that occurred 13.8 billion years ago, now called the Big Bang. Very few people, however, are aware that the "father" of this idea was a young Belgian cosmologist and Catholic priest, Fr. George Lemaittre. He formulated the theory of the "primeval atom," proposing that at the beginning, our universe could have been the same size as an atom of exceedingly high energy density. The subsequent expansion of this atom would give rise to the observed red-shifted motion of the galaxies, predicting that the rate of recessive motion observed from our vantage point should be directly related to those galaxies' distances from us... a prediction soon after confirmed by Hubble's observations.

$\bowtie \quad$ Stefano Bellucci

stefano.bellucci@1nf.infn.it

Alfio Bonanno

alfio.bonanno@inaf.it

Gabriele Gionti S. J.

ggionti@specola.va

Fabio Scardigli

fabio@phys.ntu.edu.tw

1 INFN, Laboratori Nazionali di Frascati, via E. Fermi 40, 00044 Frascati, Italy

2 INAF, Osservatorio Astrofisico di Catania, via S.Sofia 78, 95123 Catania, Italy

3 INFN, Sezione di Catania, via S. Sofia 64, 95123 Catania, Italy

4 Specola Vaticana, 00120 Vatican City, Holy See (Vatican City State)

5 Vatican Observatory Research Group, Steward Observatory, The University of Arizona, 933

North Cherry Avenue, Tucson, AZ 85721, USA

6 Dipartimento di Matematica, Politecnico di Milano, Piazza Leonardo da Vinci 32, 20133 Milan, Italy 
Lemaître was also responsible for several other notable ideas, including the discovery of the apparent singularity of the Schwarzschild metric at the event horizon, and the negative pressure generated by the cosmological constant. He served president of the Pontifical Academy of Sciences from 1960 until his death in 1966. The character and contributions of this outstanding man inspired the form of this special issue, whose topics can largely be considered to be his scientific legacy.

The contents have been significantly shaped by the recent detections of gravitational waves, which have opened a new window for our understanding of the universe. They have also been fostered by recent speculations on the true nature of the space-time singularities that signal the breakdown of classical physics, and the need for a new physics to operate at the beginning of our universe and at the end of gravitational collapse. The questions focus on the limits of modern cosmology and the scientific challenges that can be explored in the near future.

A central topic to emerge from these contents is the nature of the loss of unitarity evolution inside a Black Hole. Several different approaches to Quantum Gravity theories, such as Asymptotic Safety, Loop Quantum Gravity and String Theory are discussed and compared. Some attempts at Quantum Gravity theories show that the central singularity of Quantum Black Holes (i.e., making the Black Holes "Quantum") disappears in such a way that the information paradox can be overcome.

One clear result from the contents is that the idea of an early phase of Inflation is more of a general concept than a satisfactory theory; some of its implications such as multiverses were seriously challenged. In spite of many remaining questions, such as fine tuning and cosmological coincidence, the standard model of cosmology $(\Lambda \mathrm{CDM})$ appears to be the most accurate theory available today in cosmology to fit the observational data; but it needs two exotic ingredients: Cold Dark Matter and Dark Energy. The very nature of Cold Dark Matter is an important theme of this special issue. Its "supersymmetric" nature appears to remain in question, while the debate continues as to whether it is a particle or something completely different.

Another hotly debated topic is the measure of the Hubble constant: attempts to measure it by direct and indirect methods give two different results.

The Conformal Cyclic Cosmology proposal deserves special mention. It attempts to solve the entropy problem of the universe; it does not need the Inflation mechanism and there is no beginning of the universe. It does account for the Cold Dark Matter and for the unitarity of loss into a Black Hole.

Of course, Gravitational Waves are another central theme of this collection. While they are a natural prediction of Einstein's General Theory of Relativity, they have only recently been detected. They open a new window in modern cosmology and astrophysics, providing the possibility of detecting new phenomena as well as new ways of observing our universe.

The idea for this special issue was born during a workshop held in the beautiful pontifical Villas of Castel Gandolfo, home of the Vatican Observatory. This was the setting for a gathering from May 9 to 12, 2017 of forty scientists coming from Europe, America, Asia, and Africa to discuss "Black Holes, Gravitational Waves and SpaceTime Singularities." In order to set the scientific framework of the workshop, a number of renowned scientists gave invited talks. This workshop honored the person and the scientific legacy of Mons. George Lemaître, 50 years after his death. In addition, on 
the last day of the workshop all the participants had the privilege of being received by Pope Francis in a private audience in Vatican City.

The editors thank all authors who contributed to making this publication possible and hope, in the near future, to organize a follow-on to this workshop at the Vatican Observatory. 